\title{
ESTUDO TRANSVERSAL COM ESTUDANTES DE PSICOLOGIA SOBRE CONCEITOS DE AVALIAÇÃO PSICOLÓGICA
}

\section{Cross study with psychology students of conœpts of psychological assessment}

\author{
Ana Paula Porto Noronhaa, Fabián Javier Marín Ruedab \\ Mariana Varandas Camargo de Barros ${ }^{\mathrm{c}}$, Alexandre José Raad ${ }^{\mathrm{d}}$
}

a D outora em Psicologia Ciênciae Profissão pela Pontifícia Universidade Católica deCampinas, D ocente do Programa de PósGraduação Stricto Sensu em Psicologia da Universidade São Francisco, Bolsista Produtividade em Pesquisa do CNPq, Itatiba, SP - Brasil, e-mail: ana.noronha@ saofrancisco.edu.br

${ }^{\text {b }}$ D outorando em avaliação Psicológica pelo Programade Pós-G radução Stricto Sensu em Psicologia da Universidade São Francisco, Bolsista CAPE S, Itatiba, SP - Brasil, e-mail: marinfabian@ gmail.com

c Acadêmica do Curso de Psicologia da Universidade São Francisco, Bolsista IC/ FAPESP, Itatiba, SP - Brasil, e-mail: mariana_varandas@yahoo.com.br

d Professor do curso de Psicologia da Universidade Tiradentes, Aracaju, SE - Brasil, e-mail: araad@ unit.com.br

\section{Resumo}

O estudo sobre a formação do psicólogo na área de Avaliação Psicológica no Brasil é o tema dessa investigação. Essa pesquisa visou analisar a importância e o domínio que estudantes de Psicologia, dos cinco anos do curso, atribuíram aos conteúdos relacionados à Avaliação Psicológica. Participaram 257 jovens, com média de idade de 22,9 anos, sendo 78,2\% mulheres que cursavam do primeiro ao quinto ano, de uma universidade do Estado de Sergipe. Foi aplicado um questionário com 40 itens, baseado nas especificações do Tests $U$ sers $Q$ ualification. Os resultados apresentaram que os alunos do $1^{0}$ ano atribuíram menor importância às questões, que os de $5^{\circ}$ ano, e com relação ao domínio, os estudantes se percebem mais confiantes quando se aproximam do final da formação.

Palavras-chave: Avaliação psicológica. Formação professional. Testes psicológicos. 


\begin{abstract}
The theme of this research is the psychologist formation in Psychological Assessment in Brazil. This research analyzed the importance and domain of psychology students, in five years of regular course, attributed the contents related to Psychological Assessment. 257 students participated, mean 22,9 years old, being that 78,2\% were women studying from the first to the fifth year, from an University of Sergipe State. The questionnaire was applied with 40 items, based on specifications of Tests Users Qualification. The results presented that students of the first year attributed less importance than the fifth year to the questions, and regarding to the domain, the students feel more confident when the conclusion of graduation is coming.
\end{abstract}

Keywords: Psychological assessment. Psychologist formation. Psychological tests.

\section{FUNDAMENTAÇ̃̃O TEÓRICA}

A formação profissional do psicólogo tem sido repensada desde que o primeiro curso foi oficializado no país. Nesse sentido, tendo como meta central a formação para a atuação profissional e para a pesquisa, a Resolução n 08 do Conselho Nacional de Educação (CNE, 2004) instituiu diretrizes curriculares para os cursos de graduação em Psicologia. O objetivo foi o de repensar a preparação profissional, fundamentando-se nas habilidades, competências econhecimentos em torno de eixos estruturais. D e acordo com Bastos (2002) a proposta prevê que os cursos se diferenciariam em razão da vocação ou demandas sociais, regionais e locais, os chamados "perfis de formação". O aluno teria mais possibilidades de fazer escolhas, por meio das ênfases curriculares, ou seja, dos espaços de aprofundamentos teórico-práticos.

Tal como preconizado por Bock (1999, 2005), existem muitas 'formações' possíveis para os psicólogos, porém, a Psicologia ainda permanece na vertente tradicional, apresentando problemas, como a preocupação com o ensino de técnicas afastadas da realidade e da ética; a falta de empenho em pesquisas; a relação autoritária do professor frente ao aluno e o ensinamento de 'como aplicar' e não de 'por que aplicar'.

Bettoi e Simão (2002) assinalam a necessidade de se pesquisar cursos de formação em Psicologia para, assim, haver conhecimento crítico sobre as atividades que formarão os futuros profissionais. Maluf (1996) realizou uma pesquisa sobre a formação profissional do psicólogo no Brasil por meio de 93 entrevistas com profissionais das áreas de clínica, organizacional, educacional, social, esportiva e jurídica, intencionalmente selecionados. Em meio às conclusões, a autora acredita ser necessária a superação dos limites da formação tecnicistapara, assim, garantira formação básica do profissional antes de sua especialização.

No que se refere à avaliação psicológica, segundo Noronha e Alchieri (2004), o ensino está sendo considerado um problema central na formação do psicólogo, considerando-se que parte da responsabilidade por atuações profissionais impróprias se deva a uma formação inconsistente na área. Essa perspectiva também é compartilhada por Pereira e Carellos (1995), uma vez que as autoras já falavam sobre o ensino e a transmissão das teorias, técnicas e práticas dos exames psicológicos deficitários. Sendo assim, o professor deve fazer com que seu aprendiz conheça a fundamentação teórica subjacente ao teste psicológico, desenvolva habilidades relativas à aplicação, correção e análise e tenha acesso a uma prática efetiva e supervisionada, para que aconteça um bom ensino e a formação de um profissional competente.

O Código de Ética da A merican Psyhoological A ssociation (APA) preconizaqueospsicólogos devem oferecer serviços e usar técnicas nas quais tenham domínio. Ao lado disso, devem ter critérios para escolha dos testes, levando em consideração a pertinência do construto avaliado para o processo e as qualidades psicométricas do instrumento, a saber, sua validade e precisão, e os dados normativos (Almeida, 1999; Anastasi \& Urbina, 2000; Noronha \& Freitas, 2005).

No que serefereà escolhadosinstrumentos a serem usados, Alchieri, Noronha e Primi (2003) afirmam que esta é feita sem critério, uma vez que os profissionais desconhecem os requisitos básicos 
de uso, correção e interpretação. Para os autores, 0 ensino de instrumentos de avaliação psicológica está limitado ao contexto da graduação, de tal modo que é preciso desenvolver no país a cultura de aprimoramento contínuo e, por conseguinte, de acesso a novos dados de pesquisas.

Com relação ao ensinamento de testes psicológicos, Sbardelini (1991) e Simões (1999) sugerem que estudantes de avaliação psicológica devem possuir domínio das principais técnicas, métodos e instrumentos de avaliação, incluindo 0 conhecimento dos manuais, condições deaplicação e correção de dados de natureza psicométrica. Nesse sentido, o papel do professor, é levar 0 aluno a entender como o instrumento em questão poderá servir para que ele compreenda concretamente a pessoa que terá diante de si. Para que isto seja possível, o aluno deve ter raciocínio crítico, uma vez que o processo de avaliação inclui competências como descrever, analisar, comparar, inferir, formular hipóteses, traduzir e comunicar os resultados de forma apropriada.

No Brasil, pesquisas foram realizadas com 0 intuito de analisar aspectos diversos da formação em Psicologia. Mais especialmente, as investigações a respeito da formação em avaliação psicológica, contam com a opinião de estudantes de Psicologia, ou ainda, com a percepção deles sobre o processo de ensino-aprendizagem. A seguir são apresentados alguns trabalhos, em ordem cronológica.

Com o objetivo de criar condições que favoreçam a assimilação dos conteúdos, Primi e Munhoz (1998) realizaram um estudo com estudantes que cursavam disciplinas de TEP. Em meio às conclusões finais, os autores pontuaram que a habilidade nesse tipo de disciplina depende da motivação e participação do aluno, de suas frequências em aula, capacidade de raciocínio.

Comvistasainvestigaro reflexo dasatividades práticas realizadas na disciplina de TEP, Lima (1999) fez uma pesquisa com 150 alunos de graduação em Psicologia, aprovados na sequência destas disciplinas do $4^{0}$ ao $8^{\circ}$ semestre. 0 autor assinala que as atividades práticas da disciplina de T.E.P. sobrecarregam 0 aluno de Psicologia e não desempenham plenamente seu papel de simulação de uma situação real. Porém, tais atividades práticas, quando alicerçadas em uma fundamentação teórica previamente apresentada e discutida com os alunos, podem ser a uma excelente opção para a qualidade cada vez maior no processo ensino-aprendizagem de T.E.P.
Levando em consideração que a estatística é parte da formação do psicólogo, Silva, Britto, Carzola e Vendramini (2002), realizaram um estudo com 330 estudantes, sendo 80,2\% mulheres e 44,5\% daárea de humanas, provenientes de distintos cursos, num nível introdutório das disciplinas de estatística e matemática. Os alunos apresentaram atitudes negativas tanto em relação à estatística, quanto à matemática. Por fim, as autoras advertem que a disciplina deve ser ensinada de forma interessante aos alunos, utilizando exemplos específicos.

Noronha, Baldo, Barbin e Freitas (2003) realizaram um estudo com 180 estudantes do primeiro e do último do curso de Psicologia, no qual eles responderam à 45 questões sobre 0 conceito em avaliação psicológica, a aprendizagem e 0 uso de instrumentos, a fim de investigar seu conhecimento. Os resultados não eram esperados, uma vez que os alunos do começo do curso tiveram melhores resultados (20\%) em determinadas questões não muito sofisticadas, que os alunos do último ano.

Com o intuito de conhecer as características dos alunos de um curso de Psicologia, objetivando uma melhoria das estratégias para minimizar variáveis negativas relacionadas à formação, Baptista, A madio, Rodrigues, Santos e Palludetti (2004) realizaram um estudo com 101 alunos do $3^{\circ}$ e $4^{\underline{0}}$ ano de uma universidade particular. A coleta de dados procedeuse por meio da análise de 28 questões de um questionário. Entre os resultados, pode-se observar que $68 \%$ dos alunos têm o hábito de ler os textos antes das aulas, $51 \%$ gastam até 2 horas por semana com leituras extraclasse, 89\% estudam antes das avaliações, 40\% esclarecem dúvidas em sala, 59\% do3ํano e $76 \%$ do $4^{0}$ ano já têm linha teórica definida e 82\% pretende fazer pós-graduação.

Em um estudo sobre a formação na área de avaliação, Paula, Pereira e Nascimento (2007) abordaram 358 alunos de Psicologia que cursavam o quinto ano da graduação de quatro instituições particulares. 0 instrumento era dividido em três partes, sendo eles, formação acadêmica, articulação entre teoria e prática e identificação dos problemas mais frequentes no uso de testes psicológicos. Por fim, as autoras concluíram que, as universidades não oferecem capacitação e treinamento adequados para a prática de avaliação psicológica.

A fim de investigar a importância conferida ea percepção de domínio de estudantes de Psicologia quanto às competências de avaliação psicológica, 
Noronha, Nunes e Ambiel (2007) aplicaram 112 questionários, sendo $71 \%$ do Centro-0 este. Referente às questões analisadas, os alunos disseram que os princípios éticos, a comunicação dos resultados, o conhecimento de psicopatologia e 0 uso e a interpretação dos testes são os mais importantes, sendo que as noções de estatística e 0 conhecimento de diversos testes não são tão importantes. No que diz respeito à outra categoria, a amostra aparentou dominar os princípios éticos, a leitura dos manuais na íntegra, as condições adequadas de testagem e a utilização de tabelas dos manuais. Já 0 conhecimento de vários testes, a psicopatologia, a elaboração de parecer e 0 conhecimento dos construtos não são aspectos de domínio dos alunos. Por fim, os autores consideram importante que outros estudos sejam realizados para averiguar as competências em avaliação psicológica.

Em síntese, tendo como foco os conteúdos que deveriam ser priorizados quando da formação na área, Noronha et al. (2002) sugeriram como prioritários: teoria damedidaepsicometria; avaliação da inteligência e da personalidade; e prática de planejamento, execução e redação de resultados. Nessa mesma direção, Hutz e Bandeira (2003), acrescentaram que deveriam ser criadas estratégias para a educação continuada e para a especialização. Ao lado disso, seria interessante o desenvolvimento de programas para a qualificação de professores.

Nesse particular, Lunenberg e Willemse (2006), com vistas a incrementar as competências dos docentes na Europa, realizaram um estudo e concluíram que embora os professores estejam participando de várias pesquisas, eles não são consumidores delas. D entre as várias considerações relevantes dos autores, destaca-se a falta de estudo e de leitura deles. Embora o trabalho não estivesse falando especialmente do professor de Psicologia, ou ainda, do psicólogo, parece que tais considerações são pertinentes também em relação a esta área.

Considerando as questões abordadas sobre a formação do psicólogo na área de Avaliação Psicológica, é apresentado o objetivo do estudo. Assim, pretendeu-se analisar a importância e 0 domínio que estudantes de Psicologia, dos cinco anos do curso, atribuíram aos conteúdos relacionados à Avaliação Psicológica.
MÉTODO

\section{Participantes}

Participaram da pesquisa 257 estudantes universitários do curso de Psicologia de uma universidade particular da cidade de A racaju, Estado de Sergipe. A idade média foi 22,96 anos ( $\mathrm{D} P=5,36$ ), sendo 0 mínimo 17 e 0 máximo, 47 anos. D estacase que 44 estudantes $(17,1 \%)$ não forneceram informações referentes a essa variável. Q uanto ao sexo, $55(21,4 \%)$ eram homens e 201 (78,2\%) mulheres. Apenas 1 indivíduo não forneceu informações referentes ao sexo.

No que diz respeito ao período em que os estudantes se encontravam, ressalta-se que participaram alunos dos cinco anos do curso, sendo $26(10,2 \%)$ do $1^{\circ}$ ano, $99(38,5 \%)$ do $2^{\circ}$ ano, do $3^{\circ}$ ano do curso foram $45(17,5 \%)$ alunos, 42 (16,3\%) estavam cursando o $4^{\circ}$ ano e, por fim, 45 estudantes $(17,5 \%)$ se encontravam no último ano do curso.

\section{Material}

Foi aplicado o Questionário de Competências para Avaliação Psicológica - Q CAP (Noronha, Nunes, Ambiel \& Barros, 2007), no qual os participantes são solicitados a analisar 20 aspectos relacionados à avaliação psicológica, informando: 1) a "Importância" da atividade especificada no item e, 2) o seu "D omínio" para realizar tal atividade. Os aspectos devem ser respondidos em uma escala likert de 1 a 3, que varia entre "nada" a "muito importante" e de "nenhum" a "muito domínio" e os mesmos itens são apresentados duas vezes (uma para "Importância" e outrapara "D omínio"), totalizando 40 itens. 0 questionário foi elaborado com base nas especificações do T ests U sers Q ualification (APA, 2000) e contém aspectos que foram considerados pelos autores como relevantes para a realidade brasileira de ensino de Avaliação Psicológica.

Quanto às propriedades psicométricas do instrumento, o estudo realizado por Rueda, Noronha, Raad e Barros (2008) verificou, por meio da análise fatorial, que tanto os aspectos relacionadosà "Importância" quanto ao "D omínio" 
da avaliação psicológica formaram três fatores. No caso da Importância eles explicaram 44,89\% da variância e, no caso do Domínio, os três fatores explicaram 57,99\%.

Quanto à importância, 0 Fator 1 foi denominado "Aplicação da avaliação psicológica" e ficou composto por 13 aspectos; o Fator 2 (denominado "Correção e interpretação dos testes psicológicos") agrupou 4 aspectos e, por fim; 0 Fator 3 congregou 3 aspectos e foi denominado "Construção de testes psicológicos". Os mesmos fatores foram formados quando considerado 0 D omínio da avaliação psicológica, porém, houve a inversão dos fatores 2 e 3 , assim como também houve diferença na quantidade de aspectos que integrou cada fator. A ssim, o Fator 1 ficou composto por 10 aspectos, o Fator 2 agrupou 8 e, finalmente, o Fator 3 congregou 7 aspectos do Domínio da avaliação psicológica.

Quanto aos índices de precisão, os autores estudaram os coeficientes de precisão pelo alfa de Cronbach para cadafator edeformageral, obtiveram resultados que variaram de 0,62 a 0,93. A análise detalhada desses coeficientes permitiu afirmar que esses valores poderiam ser considerados excelentes (Arias, 1996; Conselho Federal de Psicologia, 2003), uma vez que o coeficiente de 0,62 foi obtido pelo Fator 3 da Escala de Importância, o qual ficou composto por apenas três aspectos.

\section{Procedimentos}

Após a aprovação do projeto pelo Comitê de Ética em Pesquisa de uma instituição de ensino superior e a assinaturaao Termo deConsentimento Livre e E sclarecido por parte dos respondentes ou dos seus responsáveis legais, no caso dos alunos menores de 18 anos, procedeu-se à aplicação do questionário. 0 mesmo foi aplicado coletivamente em sala de aula, com o máximo de 30 alunos por sala. 0 tempo deaplicação não excedeu 15 minutos.

\section{RESULTADOS}

Para análise dos resultados, primeiramente foram verificadas as estatísticas descritivas de cada fator e das escalas totais da Importância e do
D omínio de avaliação psicológica e, num segundo momento, foram verificadas possíveis diferenças para cada fator e para as escalas totais em função do ano que os estudantes estavam cursando. Na Tabela 1 são apresentadas as pontuações médias, mínimas, máximas e os desvios-padrão de cada fator e das escalas totais.

TABELA 1 - Estatísticas descritivas dos Fatores e das pontuações totais do Q CAP $(\mathrm{N}=257)$

\begin{tabular}{|c|c|c|c|c|}
\hline & $\mathbf{M}$ & DP & Mín & Máx \\
\hline $\begin{array}{l}\text { Importância da } \\
\text { aplicação daavaliação } \\
\text { psicológica(F1) }\end{array}$ & 35,02 & 4,20 & 13 & 39 \\
\hline $\begin{array}{l}\text { Importância da utilização } \\
\text { de um teste na avaliação } \\
\text { psicológica(F2) }\end{array}$ & 10,40 & 1,75 & 4 & 12 \\
\hline $\begin{array}{l}\text { Importância da construção } \\
\text { detestes psicológicos(F3) }\end{array}$ & 7,09 & 1,46 & 3 & 9 \\
\hline Importância total & 52,50 & 6,25 & 26 & 60 \\
\hline $\begin{array}{l}\text { D omínio da aplicação da } \\
\text { avaliação psicológica(F1) }\end{array}$ & 20,15 & 5,50 & 10 & 30 \\
\hline $\begin{array}{l}\text { Domínio da construção } \\
\text { de testespsicológicos(F2) }\end{array}$ & 14,86 & 4,06 & 8 & 24 \\
\hline $\begin{array}{l}\text { D omínio da utilização de } \\
\text { um teste na avaliação } \\
\text { psicológica(F3) }\end{array}$ & 13,08 & 3,59 & 7 & 21 \\
\hline Domínio total & 38,73 & 9,70 & 20 & 60 \\
\hline
\end{tabular}

PelaTabela 1 algumas informações podem ser verificadas. Uma delas é que em todos os fatores e nas escalas totais foram obtidas as pontuações mínimas e máximas possíveis. Também, verificouse que no caso da Importância total, a pontuação média foi bastante alta $(52,5$ de um total de 60 pontos possíveis), o que evidencia que os alunos, de forma geral, atribuem umaimportância considerável aos aspectos do Q CAP para a realização da avaliação psicológica. 
Noronha A. P. P., Rueda F. J. M., Barros M. V. C. de, Raad A. J.

Para verificar possíveis diferenças no QCPA em razão do ano que os alunos estavam cursando, realizou-se uma análise de variância, adotando como nível de significância 0,05. Os resultados podem ser observados na Tabela 2.

TABELA 2 - Valores de F e p obtidos pela análise de variância no Q CAP em função do ano do curso

\begin{tabular}{|c|c|c|}
\hline & $\mathbf{F}$ & $\mathbf{p}$ \\
\hline $\begin{array}{l}\text { Importânciada aplicação da } \\
\text { avaliação psicológica(F1) }\end{array}$ & 17,16 & 0,000 \\
\hline $\begin{array}{l}\text { Importância da utilização de um } \\
\text { testena avaliação psicológica(F2) }\end{array}$ & 10,99 & 0,000 \\
\hline $\begin{array}{l}\text { Importância da construção de } \\
\text { testes psicológicos(F3) }\end{array}$ & 3,98 & 0,004 \\
\hline Importância total & 17,22 & 0,000 \\
\hline $\begin{array}{l}\text { D omínio da aplicação da } \\
\text { avaliação psicológica(F1) }\end{array}$ & 22,93 & 0,000 \\
\hline $\begin{array}{l}\text { Domínio da construção de } \\
\text { testes psicológicos(F2) }\end{array}$ & 18,59 & 0,000 \\
\hline $\begin{array}{l}\text { D omínio da utilização de um } \\
\text { testena avaliação psicológica(F3) }\end{array}$ & 14,57 & 0,000 \\
\hline Domínio total & 21,72 & 0,000 \\
\hline
\end{tabular}

Pela Tabela 2 pode ser verificado que todos os fatores, bem como as pontuações totais, apresentaram diferenças estatísticas significativas, indicando que a importância e o domínio dos alunos quanto aos aspectos da avaliação psicológica apresentados pelo QCPA seria diferente em função do momento do curso. A prova de Tuk ey foi utilizada para explicar essas diferenças. As Tabelas 3, 4, 5 e 6 apresentam os resultados dos fatores "Importância da aplicação da avaliação psicológica”, "Importância da utilização de um teste na avaliação psicológica", "Importância da construção de testes psicológicos" e "Importância total", respectivamente.
TABELA 3 - Subconjuntos formados pela prova de Tukey em razão do ano do curso na Importância da aplicação da avaliação psicológica

\begin{tabular}{lllll}
\hline & \multicolumn{4}{c}{ Subgrupos para alfa $=\mathbf{0 , 0 5}$} \\
Ano do Curso & N & $\mathbf{1}$ & $\mathbf{2}$ & $\mathbf{3}$ \\
\hline 1 & 26 & 30,15 & \\
2 & 99 & & 34,31 & \\
3 & 45 & & 36,13 & 36,13 \\
5 & 45 & & & 36,56 \\
4 & 42 & & & 36,83 \\
\hline $\mathrm{p}$ & & 1,000 & 0,166 & 0,910 \\
\hline
\end{tabular}

TABELA 4 - Subconjuntos formados pela prova de Tukey em razão do ano do curso na Importância da utilização de um teste na avaliação psicológica

\begin{tabular}{lccc}
\hline & \multicolumn{3}{c}{ Subgrupos para alfa = 0,05 } \\
Ano do Curso & N & $\mathbf{1}$ & $\mathbf{2}$ \\
\hline 1 & 26 & 8,69 & \\
2 & 99 & & 10,17 \\
3 & 45 & & 10,76 \\
4 & 42 & & 10,88 \\
5 & 45 & & 11,07 \\
\hline $\mathrm{p}$ & & 1,000 & 0,084 \\
\hline
\end{tabular}

TABELA 5 - Subconjuntos formados pela prova de Tuk ey em razão do ano do curso na Importância da construção de testes psicológicos

\begin{tabular}{llcc}
\hline & \multicolumn{3}{c}{ Subgrupos para alfa $=\mathbf{0 , 0 5}$} \\
Ano do Curso & N & $\mathbf{1}$ & $\mathbf{2}$ \\
\hline 1 & 26 & 6,19 & \\
3 & 45 & 3,82 & 6,82 \\
2 & 99 & & 7,18 \\
4 & 42 & & 7,33 \\
5 & 45 & & 7,42 \\
\hline $\mathrm{p}$ & & 0,250 & 0,297 \\
\hline
\end{tabular}


TABELA 6 - Subconjuntos formados pela prova de Tukey em razão do ano do curso na Importância total

\begin{tabular}{lllll}
\hline & \multicolumn{4}{c}{ Subgrupos para alfa $=\mathbf{0 , 0 5}$} \\
Ano do Curso & $\mathbf{N}$ & $\mathbf{1}$ & $\mathbf{2}$ & $\mathbf{3}$ \\
\hline 1 & 26 & 45,04 & \\
2 & 99 & & 51,67 & \\
3 & 45 & & 53,71 & 53,71 \\
5 & 45 & & & 55,04 \\
4 & 42 & & 55,05 \\
\hline $\mathrm{p}$ & & 1,000 & 0,439 & 0,802 \\
\hline
\end{tabular}

De forma geral pôde ser verificado que 0 primeiro ano do curso de Psicologia apresentou as menores pontuações, sendo diferenciados dos outros anos nos três fatores da Importância e na Importância total. Ou seja, pode-se dizer que os alunosingressantes percebem osaspectosreferentesàavaliação psicológica como sendo menosimportantes do queaqueles alunos quejátiveram um contato com a avaliação psicológica, mesmo que esse contato tenha sido pequeno.

Quanto à diferenciação dos alunos que se encontram cursando o $2^{-}$ano em diante, percebeu-se que, no caso da "Importância da utilização de um teste na avaliação psicológica” , nenhum dos anos cursados foi diferenciado. Já na "Importância da aplicação da avaliação psicológica”, observou-se que os alunos dos últimos dois anos do curso ( $4^{\circ}$ e $5^{0}$ ano) foram diferenciados dos alunos do $2^{\underline{0}}$ ano, que, por sua vez, também se diferenciaram do $1 \stackrel{0}{\circ}$. No caso dos alunos que se encontravam cursando o $3^{0}$ ano, estes não foram diferenciados dos alunos do $2^{\circ}, 4^{\circ}$ e $5^{\circ}$ ano, o que permitiria inferir que seria a partir desse ano que os alunos tomariam uma maior consciência quanto à importância da utilização da avaliação psicológica.

$\mathrm{Na}$ 'Importấncia da construção de testes psicológicos' observou-se que os alunos foram separados em dois grupos, sendo que o $3^{0}$ ano não se diferenciou do restante. Por fim, quanto à "Importância total" foi observada a mesma ocorrência da "Importância da aplicação da avaliação psicológica", ou seja, a partir do 3o ano essa importância começaria a ganhar destaque.

Quanto ao Domínio, foi realizado o mesmo procedimento estatístico explicado anteriormente. Os resultados podem ser observados nas Tabelas 7, 8, 9 e10.
TABELA 7 - Subconjuntos formados pela prova de Tuk ey em razão do ano do curso no Domínio da aplicação da avaliação psicológica

\begin{tabular}{llllll}
\hline & \multicolumn{5}{c}{ Subgrupos para alfa $=\mathbf{0 , 0 5}$} \\
Ano do Curso & $\mathbf{N}$ & $\mathbf{1}$ & $\mathbf{2}$ & $\mathbf{3}$ & $\mathbf{4}$ \\
\hline 2 & 99 & 17,38 & & \\
1 & 26 & 18,15 & 18,15 & & \\
3 & 45 & & 20,64 & 20,64 & \\
4 & 42 & & 22,31 & 22,31 \\
5 & 45 & & & 24,87 \\
\hline $\mathrm{p}$ & & 0,944 & 0,112 & 0,485 & 0,096 \\
\hline
\end{tabular}

TABELA 8 - Subconjuntos formados pela prova de Tukey em razão do ano do curso no Domínio na construção de testes psicológicos

\begin{tabular}{lllll}
\hline & \multicolumn{4}{c}{ Subgrupos para alfa $=\mathbf{0 , 0 5}$} \\
Ano do Curso & $\mathbf{N}$ & $\mathbf{1}$ & $\mathbf{2}$ & $\mathbf{3}$ \\
\hline & \multicolumn{4}{c}{12,86} \\
\\
2 & 26 & 14,08 & 14,08 \\
2 & 99 & & 15,33 & \\
3 & 45 & 16,07 & 16,07 \\
4 & 42 & & 18,11 \\
5 & 45 & 0,519 & 0,079 & 0,069 \\
\hline $\mathrm{p}$ & & & & \\
\hline
\end{tabular}

TABELA 9 - Subconjuntos formados pela prova de Tuk ey em razão do ano do curso no D omínio na utilização de um teste na avaliação psicológica

\begin{tabular}{llllll}
\hline & \multicolumn{5}{c}{ Subgrupos para alfa = $\mathbf{0 , 0 5}$} \\
Ano do Curso & $\mathbf{N}$ & $\mathbf{1}$ & $\mathbf{2}$ & $\mathbf{3}$ & $\mathbf{4}$ \\
\hline 1 & 26 & 10,81 & & \\
2 & 99 & 11,98 & 11,98 & \\
3 & 45 & & 13,02 & 13,02 & \\
4 & 42 & & & 14,45 & 14,45 \\
5 & 45 & & & & 15,60 \\
\hline $\mathrm{p}$ & & 0,458 & 0,577 & 0,255 & 0,480 \\
\hline
\end{tabular}


TABELA 10 - Subconjuntos formados pela prova de Tukey em razão do ano do curso no Domínio total

\begin{tabular}{|c|c|c|c|c|c|}
\hline \multirow[b]{2}{*}{ Ano do Curso } & \multirow[b]{2}{*}{$\mathbf{N}$} & \multicolumn{4}{|c|}{ Subgrupos para alfa $=\mathbf{0 , 0 5}$} \\
\hline & & & 2 & 3 & 4 \\
\hline 2 & 99 & 34,15 & & & \\
\hline 1 & 26 & 34,77 & 34,77 & & \\
\hline 3 & 45 & & 39,40 & 39,40 & \\
\hline 4 & 42 & & & 42,31 & 42,31 \\
\hline 5 & 45 & & & & 47,07 \\
\hline $\mathrm{p}$ & & 0,997 & 0,085 & 0,501 & 0,071 \\
\hline
\end{tabular}

Quanto ao domínio, observou-se que no caso da 'aplicação da avaliação psicológica' e a 'utilização de um teste na avaliação psicológica', os cinco anos do curso foram separados em 4 grupos, sendo que, com o passar dos anos, esse tipo de domínio aumentou. Exceção feita aos alunos do $1^{\circ} \mathrm{e} 2^{\underline{0}}$ ano no fator referente à aplicação. Já no 'Domínio na construção de testes psicológicos', o $1^{10}$ e o $5^{0}$ anos ficaram diferenciados do restante, ou seja, os alunos ingressantes apresentam maior rigor quanto às suas limitações para a construção de testes, enquanto que os alunos do $5^{0}$ ano se avaliaram com mais preparo.

Ainda no que se refere ao tema, os alunos do $2^{\circ}$ ao $4^{\circ}$ ano apresentam maior domínio que os do $1^{0}$, mas menor que os alunos do último ano. Um aspecto importante pode ser destacado, qual seja, os alunos do $3^{0}$ ano ficaram separados do restante, em um grupo intermediário, sendo que os alunos do $2^{\underline{0}}$ e do $4^{\underline{0}}$ ano ficaram misturados nesse agrupamento. Assim, o $2^{\underline{0}}$ ano ficou próximo do $1^{\underline{0}}$ e $04^{\underline{0}}$ mais próximo dosalunos do 5aano. Finalmente, no caso do "D omínio total" foi observada a mesma situação relatada no "Domínio da aplicação da avaliação psicológica" e no "D omínio na utilização de um teste na avaliação psicológica."

\section{DISCUSSÃO}

A presente pesquisa estudou a importância que alunos de Psicologia dos 5 anos de formação atribuem aos conceitos de avaliação psicológica. Ao lado disso, avaliou-se o domínio que eles possuem aos mesmos conceitos. Para que isto fosse possível, aplicou-se um questionário em alunos de Sergipe.

Os achados revelaram que, em relação à importância, claramente os estudantes dão destaque aos conceitos de avaliação psicológica, à medida que avançam no curso. Nesse sentido, observou-se que os alunos do $1^{0}$ ano atribuíram menor importância, que os de $5^{0}$ ano, embora tenha havido alguma inversão em um dos fatores. Q uanto ao domínio, os resultados em geral, permitiram identificar que os estudantes se percebem com maior domínio quanto mais se aproxima o final da formação. Em alguma medida, os resultados corroboram a pesquisa de Noronha, Baldo, Barbin e Freitas (2003). As autoras identificaram que, em geral, os alunos do começo do curso tiveram melhores resultados, que os alunos do último ano, exceto em determinadas questões.

Assim, parece que o manuscrito atendeu seu objetivo. No entanto, embora tenha se observado que estudantes ao final da graduação atribuem maior importância aos problemas da área de avaliação psicológica, a análise não pode ser encerrada aqui, uma vez que pouco colabora para com a reflexão dos problemas na formação, identificados na fundamentação desse estudo (Sbardelini, 1991; Pereira \& Carellos, 1995; Simões, 1999; Alchieri, Noronha \& Primi, 2003; Paula, Pereira \& Nascimento, 2007; dentre outros).

Uma observação relevante ainda se faz necessária. As médias atribuídas à importância foram superiores, em todos os fatores, assim como no escore total, daquelas conferidas ao domínio. Noronha, Nunes e Ambiel (2007), ao aplicarem questionários em universitários do Centro-O este do estado de São Paulo, encontram resultados semelhantes. Nesse sentido, parece interessante que os próprios alunos reconheçam que, em vários aspectos da avaliação psicológica, eles se encontram aquém do que consideram relevante.

Em síntese, as considerações de Noronha et al. (2002), na direção de que a teoria da medida e psicometria, a avaliação da inteligência e da personalidade, e prática deplanejamento, execução e redação de resultados devem ser conteúdos mais discutidos nos cursos de formação do psicólogo, parecem ainda atuais. Essas informações ao lado de outras, como a oferecida por esse estudo, devem ser consideradas quando da reformulação dos currículos em Psicologia, de tal modo que os conteúdos identificados como "carentes" possam ser mais bem trabalhados.

Por certo, este estudo não satisfez todas as necessidades de pesquisa. Os resultados aqui discutidos refletem um único estado do país e uma 
formação especifica. Assim, sugere-se que outras amostras sejam também pesquisadas.

\section{REFERÊNCIAS}

Alchieri, J. C., Noronha, A. P. P., \& Primi, R. (2003). Guia de referência: Testes Psicológicos comercializados no Brasil. São Paulo: Casa do Psicólogo.

Almeida, L. S. (1999). Avaliação psicológica exigências e desenvolvimento nos seus métodos. In S. M. Wechsler. \& R. S. L. Guzzo. Avaliação psicológica: perspectiva internacional. (pp. 41-55). São Paulo: Casa do Psicólogo.

American Psychological Association. (2000). Report of the task force on test user qualifications. Washington: American Psychological Association.

Anastasi, A., \& Urbina, S. (2000). Testagem psicológica. Porto Alegre: ArtMed.

Arias, R. M., (1996). Psicometría: Teoria de los tests psicológicos y educativos. Espanha: Sintesis Psicologia.

Baptista, M. N., Amadio, A., Rodrigues, E. C., Santos, K, M. \& Palludetti, S. A. T. (2004). Avaliação dos hábitos, conhecimentos e expectativas de alunos de um curso de psicologia. Psicol Esc Educ, 8(2), 207-217.

Bastos, A. V. B. (2002). Perfis de formação e ênfases curriculares: o que são e por que surgiram? Revista do Departamento de Psicologia, 14(1): 31-58.

Bettoi, W., \& Simão, L. M. (2002). Entrevistas com profissionais como atividade de ensinoaprendizagem desejável na formação do psicólogo. Psicologia: Reflexão e Crítica, 15(3), 613-624.

Bock, A. M. B. (1999). Atuação profissional e formação do psicólogo: os desafios da modernidade. Psicologia em Estudo, 4(1), 1-12.

Bock, A. M. B. (2005). Novo projeto para profissão no Brasil: Contribuições para a formação. Recuperado em 21 jan. 2008, da Abepsi (Abepsi on line): http:// www.abepsi.org.br/ web/ artigos.aspx
Conselho Nacional de Educação - CNE. (2004). Resolução no 8. Recuperado em 19 jan. 2008, da Abepsi (Abepsi on line): http:/ / www.abepsi.org.br/ web/ linhadotempo.aspx

Conselho Federal de Psicologia (2003). Resolução 02/ 2003. Recuperado em 21 jun. 2008, da pol (pol on line): www.pol.org.br

Hutz, C. S., \& Bandeira, D. R. (2003). Avaliação Psicológica no Brasil: situação atual e desafios para o futuro. In M. O. H. Yamamoto \& V. V. Gouveia. (O rg.). Construindo a psicologia brasileira: Desafios da ciência e prática psicológica. (pp. 261-275). São Paulo: Casa do Psicólogo.

Lima, R. A. (1999). O reflexo das atividades prática no processo de ensino-aprendizagem das técnicas de exame psicológico (p. 156-158). In Anais, 2. Encontro Sobre Psicologia Clínica, 1999. São Paulo: Universidade Presbiteriana Mackenzie.

Lunenberg, M., \& Willemse, M. (2006). Research and professional development of teacher educators. European Journal of Teacher Education, 29(1), 81-98.

Maluf, M. R. (1996). A formação profissional do psicólogo brasileiro. Interações: Estudos e Pesquisas em Psicologia, 1(1), 31-45.

Noronha, A. P., \& Alchieri, J. C. (2004). Conhecimento em avaliação psicológica. Estudos de psicologia, 21(1), 43-52.

Noronha, A. P. P., Baldo, C. R., Barbini, P. F., \& Freitas, J. V. (2003). Conhecimento em avaliação psicológica: Um estudo com alunos de psicologia. Psicologia: Teoria e Prática, 5(2), 37-46.

Noronha, A. P. P., \& Freitas, F. A. (2005). Testes psicológicos, usos e conhecimento. Psico, 36(1), 21-28.

Noronha, A. P. P., Nunes, M. F. O., \& Ambiel, R. A. M. (2007). Importância e domínios de avaliação psicológica: um estudo com alunos de Psicologia. Paidéia, 17(37), 231-244.

Noronha, A. P. P., Ziviani, C., Hutz, C. S., Bandeira, D. R., Custódio, E. M., \& Alves, I. B. 
(2002) Em defesa da avaliação psicológica. Avaliação Psicológica, 1(1), 173-174.

Noronha, A. P. P., Nunes, M. F. O., Ambiel, R. A. M., \& Barros, M. V. C. (no prelo). (2007). Questionário de competências para avaliação psicológica - QCAP.

Paula, A. V., Pereira, A. S., \& Nascimento, E. (2007). O pinião de alunos de psicologia sobre o ensino em avaliação psicológica. PsicoUSF, 12(1), 33-43.

Pereira, A. P. C., \& Carellos, S. D. M. S. (1995). Examinando o ensino das técnicas de exame psicológico. Caderno de Psicologia, 3(4), 33-36.

Primi, R., \& Munhóz, A. M. H. (1998). Um estudo sobre proficiência na disciplina de Técnicas de Avaliação Psicológica (TEP). PsicoUSF, 3(2), 75-86.

Rueda, F. J. M., Noronha, A. P. P., Raad, J. A., \& Barros, M. V. C. (no prelo). (2008). Questionário de competência em avaliação psicológica: Análise da estrutura interna.

Sbardelini, E. T. B. (1991). Os mitos que envolvem os testes psicológicos. Documenta CRP-08, 1(1), 53-57.

Silva, C. B., Brito, M. R. F., Cazorla, I. M., \& Vendramini. (2002). A titudes em relação à estatística e à matemática. Psico-USF , 7(2), 219-228.

Simões, M. R. (1999). O Ensino e a aprendizagem da avaliação psicológica: 0 caso da avaliação da personalidade. Psychologica, 22, 135-172.

Recebido: 16/ 10/ 2008

Received: 10/ 16/ 2008

Aprovado: 19/11/ 2008

A pproved: 11/ 19/ 2008 